spruchsfreie Resultate. Aus dieser Überlegung erkennt man, daß die übliche Forderung der Korrespondenz zwischen Quantentheorie und klassischer Theorie sicher nicht ausreicht, um den quantentheoretischen Formalismus festzulegen.

Noch offenbarer ist die Mehrdeutigkeit des üblichen Formalismus in der Theorie des $\mathrm{Yu}$ kawa-Feldes. Man kann hier entweder die Tatsache anerkennen, daß die Kupplung zwischen dem Yukawa-Feld und den schweren Teilchen stark ist ${ }^{19}$, wenn die Kernkräfte durch das Y ukawa-Feld erklärt werden sollen, oder man kann ein Subtraktionsverfahren anwenden, um die Glieder starker Kupplung zu beseitigen. Die beiden Möglichkeiten führen $\mathrm{zu}$ ganz verschiedenen $\eta$-Matrizen und daher auch $\mathrm{zu}$ ganz verschiedenen physikalischen Ergebnissen. Es dürfte einstweilen schwierig sein, zu entscheiden, welche Annahme den Experimenten besser entspricht. Vielleicht werden die qualitativen Züge beider Theorien in der späteren Theorie eine Rolle spielen.

19 G. W e n t z e l, l. c. ${ }^{2}$. c) Zum Schluß wollen wir versuchen, die Bedingungen zusammenfassend $\mathrm{zu}$ formulieren, die eine Theorie der Elementarteilchen erfüllen muß: Eine solche Theorie muß zu einer relativistisch invarianten unitären Matrix $S$ führen, die das asymptotische Verhalten der Wellen und die stationären Zustände in der üblichen Weise bestimmt. Der Formalismus, der $S$ festlegt, muß in der Grenze, in der die universelle Länge von der Größenordnung $10^{-13} \mathrm{~cm}$ als klein angesehen werden kann, in den üblichen Formalismus mit einer $\mathrm{H}$ amilton schen Funktion übergehen. Aber auch abgesehen von diesem Grenzfall, muß der neue Formalismus dem früheren insofern ähnlich sein, als er eine Funktion $S$ ergeben muß, die in derselben Weise „glatt" ist wie die aus gewöhnlichen Hamilton-Funktionen bestimmten $S$-Funktionen. Der richtige Formalismus liegt also wahrscheinlich irgendwo in der Mitte zwischen einem Formalismus der Art (42) und einem Schema, bei dem einfach die Matrix $\eta$ gegeben wird.

\title{
Zur Theorie des Elektrons
}

\author{
Von Walter Wessel, Graz ${ }^{1}$
}

(Z. Naturforschg. 1, 622-636 [1946]; eingegangen am 13. Juli 1946)

$I^{\prime}$ n dem Bestreben, die Reaktionskraft der Strahlung auf ein bewegtes, geladenes Teilchen in die Quantenmechanik einzubauen und so vielleicht den Schwierigkeiten der Quantenelektrodynamik zu entgehen, wurde der Verf. schon vor längerer Zeit $^{2}$ auf sehr merkwürdige Zusammenhänge zwischen Strahlungskraft und Spin geführt, die zunächst allen Deutungsversuchen widerstanden. Nach verschiedenen Bemühungen, der Sache von der klassischen ${ }^{3}$ oder der quantentheoretischen ${ }^{4}$ Seite nahezukommen, ließ sich zuletzt das Problem in eine klarere Form bringen durch die Fragestellung ${ }^{5}$, ob und welche Vertauschungsrelationen mit einem die Strahlungskraft enthaltenden System von Bewegungsgleichungen eines Elektrons vereinbar wären. Folgendes wurde erreicht:

1 z. Zt. in Heidelberg.

2 W. W e s s e l, Z. Physik 92, 407 [1934].

3 W. W e s s e l, Z. Physik 110, 625 [1938].
1. Man kann durch einen Kunstgriff von ungezwungener Einfachheit die von der bewegten Ladung ausgestrahlte Energie in der Ruhmasse mitzählen. Das System wird dann konservativ, und es lassen sich kanonische Impulse einführen. Wegen des Einflusses der Strahlungsreaktion wird der kanonische Impuls nicht nur Funktion der Geschwindigkeit, sondern auch der Beschleunigung. Impuls und Geschwindigkeit werden dadurch unabhängige Veränderliche. Diese Unabhängigkeit ist eine der wesentlichsten Eigentümlichkeiten der D i r a c schen Spintheorie.

2. Die Hamilt on sche Funktion nimmt genau die Diracsche Form an. Das ist möglich, weil darin auch bei D i r a c nur Koordinaten, Impulse und Geschwindigkeiten, dagegen keine Spinoperatoren auftreten. Da die Ruhmasse wegen der darin mitgezählten Strahlungsenergie zeitveränderlich

4 W. W e s s e l, Naturwiss. 30, 606 [1942].

5 W. W e s s e l, Ann. Physik (5) 43, 565 [1943]. 
wird, läßt sich einsehen, warum ihr bei $\mathrm{D}$ i r a c ein eigener Operator zugeordnet ist.

3. Sucht man Vertauschungsrelationen einzuführen, die mit den Bewegungsgleichungen verträglich sind, so wird man zu der Folgerung geführt, daß die Quadrate der Geschwindigkeitskomponenten gleich dem Quadrat der Lichtgeschwindigkeit sein müssen. Das ist wieder ein Charakteristikum der D i r a c schen Theorie.

Schließlich bleiben nur noch die Vertauschungsrelationen der Geschwindigkeitskomponenten untereinander übrig. Diese können bei uns nicht so einfach herauskommen wie bei Dirac, denn sonst würden sich die Theorien überhaupt nicht mehr unterscheiden. In der Tat tritt hier, aber auch erst hier, der große Unterschied zutage, der zwischen Spin und Strahlungskraft kinematisch (periodische-aperiodische Störung) und dynamisch (Größenordnung der Termstörungen im Verhältnis der Feinstrukturkonstanten) besteht. Dennoch wurden auch diese Relationen näherungsweise und dem Sinne nach erhalten; sie waren jedoch in der letzten Fassung unserer Theorie noch unter sich widerspruchsvoll.

Die Behebung dieser Schwierigkeit ist der Hauptgegenstand der vorliegenden Arbeit. Sie gelingt im Rahmen einer klassischen Theorie, in der P o is s o n-Klammern an Stelle der Vertauschungsrelationen treten. Dabei ergeben sich nun auch Gesichtspunkte zur Deutung der ganzen Erscheinung.

\section{Das Moment als selbständige} Variable

Schon am Schlusse der vorigen Mitteilung wurde angedeutet, daß die Lösung in der Einführung weiterer Variabler zu suchen sei. Hierfür spricht außer ad hoc zu findenden auch ein übergeordneter physikalischer Gesichtspunkt. Der bisher von uns benutzte Ausdruck für die Reaktionskraft der Strahlung, der zeitliche Ableitungen bis zur dritten enthielt, gilt nur für ein streng punktförmiges Elektron ${ }^{6}$. Die Entwicklung der Quantenelektrodynamik führt aber immer wieder auf die alte Notwendigkeit zurück, dem Elektron in irgendeinem Sinne die Qualität einer räumlichen Ausdehnung zu geben; der ,Elektronenradius" $e^{2} / m c^{2}$ ist die Grenze, an der. auch die

- P. A. M. D i r a c, Proc. Roy. Soc. [London], Ser. A, 167, 148 [1938]. neueren Entwicklungen aufgehalten werden. Berechnet man nun für eine im gewöhnlichen Sinne räumlich ausgebreitete Ladung die Reaktions$\mathrm{kraft}^{7}$, so findet man eine in höheren Ableitungen der Koordinaten nach der Zeit fortschreitende Reihe. Es wäre alsó durchaus verständlich, wenn die Strahlungskraft auch für ein in einem abstrakteren Sinne endlich ausgedehntes Teilchen noch höhere Ableitungen, vielleicht in endlicher Anzahl, enthielte. Höhere Ableitungen übersetzen sich aber beim Abbau der Bewegungsgleichung immer in neue Variable.

Diese Variablen werden sich aus Kovarianzgründen immer zu den Komponenten eines Vektors oder Tensors zusammenschließen, der dann als neue physikalische Eigenschaft des Teilchens in Erscheinung tritt. Damit eröffnet sich die Möglichkeit, das Moment des Elektrons als Äquivalent für seine endliche Ausdehnung aufzufassen. Man nehme nämlich einmal an, seine Bewegungsgleichung sei nicht dritter, wie beim punktförmigen Teilchen, sondern fünfter Ordnung. Beim Abbau in ein System von Gleichungen erster Ordnung erhält man $5 \cdot 3=15$, unter Einbeziehung der im oben angedeuteten Sinne veränderlichen Ruhmasse 16 Bestimmungsstücke. Davon sind 3 Koordinaten, weitere 3 Geschwindigkeitskomponenten, und 4 bilden den Energie-Impuls-Vierervektor, der die Masse mitbestimmt. Danach bleiben noch 6 übrig, und das ist gerade die Komponentenzahl eines antisymmetrischen Tensors. Eine Differentialgleichung vierter Ordnung hätte auf 13 Variable, also außer den vorhandenen auf 3 neue, geführt. Hierfür ergäbe sich keine bekannte Deutung, denn der mechanische Drall muß entweder zu einem Vierer- oder Sechservektor ergänzt werden ${ }^{8}$. Wir werden also einmal versuchen, das magnetische Moment des Elektrons (wir meinen damit immer den entsprechenden Sechservektor) als neue Variable einzuführen.

Die Diracsche Theorie kennt außer dem Moment noch eine Reihe von weiteren Variablen, deren Operatoren alle aus den bisher von uns benutzten, der Geschwindigkeit und dem Massengliede, durch Aufmultiplizieren entstehen. Es sind insgesamt 16 linear unabhängige Operatoren -

7 Siehe z. B. H. A. Lorentz, Enzykl. mathem. Wissensch. V.2. Beitrag 14. Ziff. III, oder mit neueren Hilfsmitteln: F. B opp, Z. Naturforschg. 1, 53 [1946].

${ }^{8}$ L. H. Th o m a s, Philos. Mag. J. Sci. (7) 3, 1 [1927]. 
ihre Zahl stimmt nur zufällig mit der oben vorkommenden überein - , denen der Vierervektor $j_{i}$ der Strom-Ladungsdichte, ein zweiter Vierervektor $k_{i}$, dessen drei räumliche Komponenten den Spin bilden, der Sechservektor $M_{i k}$ des magnetischen und elektrischen Momentes und zwei Invarianten entsprechen. Wenn man außer den vier Elementen, aus denen sich die übrigen aufbauen, noch weitere in die klassische Theorie übernimmt, scheint es unabweisbar, alle darin aufzunehmen. Das wird auch ganz von selbst geschehen, aber die Variablenzahl erhöht sich damit nicht entsprechend. Denn physikalische Bedeutung haben ja nicht die Operatoren - so wenig wie etwa der Nabla-Operator in der gewöhnlichen Wellenmechanik -, sondern die damit zu bildenden Matrizen oder Dichtefunktionen, also beispielsweise nicht der Transformationsoperator (vierzeilige Einsermatrix) $\alpha_{1}$ der Geschwindigkeit, sondern seine (unendliche) Matrix $\left(\alpha_{1}\right)_{m n}$ in einer geeigneten Darstellung oder die Stromdichte

$\psi^{*} \alpha_{1} \psi=\psi_{1}^{*} \psi_{4}+\psi_{2}^{*} \psi_{3}+{\psi_{3}}^{*} \psi_{2}+\psi_{4}^{*} \psi_{1}$,

und zwischen diesen Größen besteht noch eine Reihe von algebraischen Beziehungen. Sie wurden teilweise schon von $\mathrm{D}$ a $\mathrm{r}$ w i $\mathrm{n}^{9}$ entdeckt; eine vervollständigte, übersichtliche Zusammenstellung verdankt man Laporte und Uhlenbeck ${ }^{\mathbf{1 0}}$. Es ergibt sich: die zwei Invarianten sind durch die beiden Invarianten des antisymmetrischen $\mathrm{Mo}^{-}$ mententensors bestimmt; der Vierervektor $k$ ist durch den Momententensor und den Vektor $j$ mitbestimmt, und dessen Quadrat führt wieder auf die Invarianten. Das sind 7 kovariante Beziehungen, durch die sich die Zahl der Unabhängigen von 16 auf 9 vermindert. Wir werden deshalb den zeitartigen Vektor $j$ durch Division mit seinem Betrage auf einen Vektor $u=j /|j|$ von der Natur einer Vierergeschwindigkeit mit drei nunmehr unabhängigen Komponenten reduzieren; dieser in Verbindung mit dem sechskomponentigen Momententensor ergibt dann gerade 9 unabhängige Bestimmungsstücke. Darüber hinauszugehen besteht einstweilen kein Anlaß. Man kann sich ja wohl versprechen - dieser Gedanke war schon immer mitbestimmend beim Versuche dieser ganzen Theorie -, daß sich mit einem so umfang-

9 C. G. D a r w i n, Proc. Roy. Soc. [London], Ser. A, 120, 621 [1928]. reichen Apparate einiges mehr als nur das reine Spinphänomen sollte beschreiben lassen.

Die Vektoren $j$ und $k$ stehen senkrecht aufeinander und sind vom gleichen Betrage. Da $j$ zeitartig ist, wird $k$ raumartig, und wenn wir $u=j /|j|$ als eine Unterlichtgeschwindigkeit auffassen, wird $U=k /|k|$ eine Überlichtgeschwindigkeit. Als solche sollte sie keine physikalische Bedeutung haben, und in der Tat ist sie nur eine reine Rechengröße, da ja $k$ durch die anderen Variablen mitbestimmt ist. Es dient aber der Vereinfachung der Gleichungen, wenn man sie mitführt. Aus

$$
j_{i} k^{i}=0
$$

folgt, wenn man von den Vierervektoren $u_{i}$ und $U_{i}$ $\mathrm{zu}$ den gewöhnlichen Geschwindigkeiten $\mathfrak{v}$ und $\mathfrak{i}$ übergeht - die Umrechnung folgt in Abschn. 3-,

$$
\mathfrak{v} \mathfrak{B}=c^{2}
$$

( $c=$ Lichtgeschwindigkeit). Wenn $\mathfrak{b}$ und $\mathfrak{B}$ gleichgerichtet sind, verhalten sie sich also wie die Phasengeschwindigkeit der Schröding er-Wellen zur Gruppengeschwindigkeit der Teilchen. Wir werden $\mathfrak{b}$ als die Geschwindigkeit im gewöhnlichen Sinne betrachten und $\mathfrak{B}$ als die zugeordnete Geschwindigkeit bezeichnen.

\section{Grundsätze und Annahmenzum A ufbauder Theorie}

Es handelt sich nun darum, die richtigen Bewegungsgleichungen für die neueingeführten Momentkomponenten $\mathrm{zu}$ finden und die Gleichungen für die Geschwindigkeit so zu verbessern, daß die am Schlusse der vorangehenden Arbeit auftretende Symmetrieschwierigkeit vermieden wird. Der Gesichtspunkt ist dabei immer dieser: ursprüngliche Bedeutung haben nur die Koordinaten und ihre zeitlichen Ableitungen, Geschwindigkeit, Beschleunigung, Änderung der Beschleunigung usw. Insbesondere hängt die Strahlungskraft nur hiervon ab. Alle übrigen Variablen: Impulse, Momente und Invarianten, stehen nur als Vertreter dieser Ableitungen zum Zwecke der Einführung von Vertauschungsrelationen. Ihre Bewegungsgleichungen müssen also so bestimmt werden, daß durch ihre Elimination die Bewegungsgleichung

${ }^{10}$ O. La p orte u. G. E. Uhl enbeck, Physie. Rev. 37, 1380 u. 1552 [1931]. 
des Teilchens unter dem Einflusse der Strahlungskraft entsteht ${ }^{11}$.

Dies Problem ist freilich noch wenig definiert. Erstens kennt man aus Versuchen die Strahlungsdämpfung höchstens mit derjenigen Genauigkeit, wie sie dem Ersatz des Elektrons durch ein punktförmiges Teilchen entspricht, und es wird genügen, wenn die verallgemeinerte Strahlungskraft in erster Näherung damit übereinstimmt. Aber auch diese Übereinstimmung braucht nicht vollständig zu sein, denn die Reaktionskraft enthält außer dem Energie verzehrenden, irreversiblen Bestandteile noch andere, reversible ${ }^{12}$, die sich im Laufe der Bewegung herausmitteln. Es genügt offenbar, wenn die irreversiblen Bestandteile in erster Näherung richtig herauskommen.

Wir müssen also von anderen Gesichtspunkten ausgehen und werden die Frage der Quantisierbarkeit unserer Gleichungen in den Vordergrund stellen. Quantenbedingungen bedeuten Vertauschungsrelationen, und Vertauschungsrelationen entsprechen in der klassischen Theorie $\mathrm{P}$ o i s s on Klammern. Durch diese kann man bekanntlich die Bewegungsgleichungen aus einer $\mathrm{H}$ a miltonschen Funktion ableiten. Wir stellen uns also die Aufgabe, ein System von Poisson-Klammern und eine $H$ a milt o n sche Funktion zu finden, so, $d a \beta$ die Bewegungsgleichungen den im Vorangehenden erhobenen Forderungen genügen. Die vorliegende Arbeit führt dieses Programm auf Grund einfacher Annahmen durch bis zur Aufstellung der Bewegungsgleichungen und einem Ausblick auf die Form der Strahlungsreaktion. Ihre genaue Bestimmung erfordert sehr weitläufige Eliminationsrechnungen, wobei dann noch die Abtrennung der reversiblen Bestandteile ein Problem für sich bleibt. Wir begnügen uns daher mit einer mehr physikalischen Betrachtung zur Möglichkeit einer nicht-konservativen Quantenmechanik, die durch das Auftreten zweier Ge-

11 Bewegungsgleichungen eines Teilchens mit magnetischem Moment wurden schon von mehreren Autoren aufgestellt. Unserm Problem am nächsten steht eine Arbeit von H. J. Bhabha u. H. C. Corben, Proc. Roy. Soc. [London], Ser. A, 178, 273 [1941], in der die Bewegungsgleichungen nur aus den Forderungen der Erhaltung von Energie und Drehimpuls des Teilchens und des Feldes, also unter Berücksichtigung der Strahlungsreaktion abgeleitet werden. Diese Autoren rechnen jedoch mit einem - der Ladung nach punktförmigen - Teilchen, dem sie zusätzlich Trägheitsmoment und Drehimpuls beilegen. Sie haben daher, wie in der Mechanik starrer Körper, von einer schwindigkeiten eine interessante, neue Gestalt annimmt.

Man hätte wohl kaum eine Handhabe, diese Aufgabe überhaupt anzugreifen, wenn nicht der enge Zusammenhang zwischen unserem Problem und der D i r a c schen Theorie vorläge. Er liefert vor allem eine naheliegende Antwort auf die Frage, welche kanonischen Variablen der Bildung der Poisson-Klammern zugrunde gelegt werden sollen. Unter einer $\mathrm{P}$ o is s o n - Klammer versteht man bekanntlich folgendes: Gegeben sei ein System von kanonischen Veränderlichen $p_{1} \ldots p_{F}$, $q_{1} \ldots q_{F}$ und zwei Funktionen $f$ und $g$ dieser Veränderlichen. Dann ist ${ }^{13}$ die P o is s o n-Klammer von $f$ und $g$ :

$$
(f, g)=\sum_{k=1}^{F}\left(\frac{\partial f}{\partial p_{k}} \frac{\partial g}{\partial q^{k}}-\frac{\partial f}{\partial q^{k}} \frac{\partial g}{\partial p_{k}}\right)
$$

Insbesondere ist also

$$
\left(p_{i}, q^{k}\right)=\delta_{i}^{k} .
$$

Im Gegensatz zu Vertauschungsrelationen, die sich frei bilden lassen, setzt also die Bildung von P o isson-Klammern ein System von kanonischen Variablen voraus.

Wir stehen vor der Aufgabe, für den Vierervektor $j /|j|$ und den Momententensor $M_{, k}$ Pois son-Klammern $\mathrm{zu}$ finden. Beide drücken sich bilinear in Spinoren $\psi, \chi$ aus: es ist z. B. (vergl. die erstgenannte Arbeit von Laporte u. Uhlenbeck ${ }^{10}$ )

$$
j_{\dot{m} l}=\psi_{\dot{m}} \psi_{l}+\chi_{\dot{m}} \chi_{l} .
$$

Unter den $\psi, \chi$ sind dabei zunächst Koordinatenfunktionen verstanden; $j$ ist eine räumliche Dichte. Es liegt nun sehr nahe, auch das klassische Elektron einmal durch vier Spinoren $\psi_{1}, \psi_{2}, \chi_{1}, \chi_{2}$ und ihre komplex Konjugierten $\psi_{1}, \psi_{\dot{2}}, \chi_{i}, \chi_{2}$ zu kennzeichnen und diese, nicht mehr als Koordinaten-

Schwerpunkts- und einer Momentengleichung auszugehen. Wir halten uns demgegenüber genau an die Variablen der D i r a c schen Theorie, haben dementsprechend kein Trägheitsmoment des Teilchens, noch auch den Drall als selbständige Variable und können grundsätzlich alles auf eine Gleichung für die Schwerpunktskoordinaten zurückführen.

${ }_{12}$ G. A. S c h o t t, Philos. Mag. J. Sci. (6) 29, 49 [1915].

${ }_{13}$ Wir definieren hier, um bei der Bildung von Bewegungsgleichungen die in der Quantenmechanik übliche Faktorenfolge zu haben, mit anderem Vorzeichen als üblich. 
funktionen, sondern als zusätzliche Bewegungsparameter betrachtet, neben den gewöhnlichen Koordinaten und Impulsen als kanonische $\mathrm{Va}$ riable einzuführen. Es seien etwa $\psi$ und $\chi$ kanonisch konjugiert, es gelte also

$$
\begin{aligned}
& \left(\psi_{\mu} \chi^{\nu}\right)=\delta_{\mu}^{\nu} \\
& \left(\psi_{\mu} \chi^{\nu}\right)=\delta_{\mu}^{\cdot \dot{v}}
\end{aligned}
$$

während alle übrigen Klammern verschwinden. Setzt man nun den Vektor $j_{\dot{m} l}$ genau wie unter (2.3) zusammen, so lassen sich sofort die $\mathrm{P}$ o is s o n - Klammern seiner Komponenten bilden nach der Formel

$$
\begin{aligned}
\left(j_{\dot{m} k} j_{\dot{n}}\right) & =\sum_{\lambda}\left(\frac{\partial j_{\dot{m} k}}{\partial \psi_{\lambda}} \frac{\partial j_{\dot{n} l}}{\partial \chi_{i}^{\lambda}}-\frac{\partial j_{\dot{m} k}}{\partial \chi^{\lambda}} \frac{\partial j_{\dot{n} l}}{\partial \psi_{\lambda}}\right) \\
& +\sum_{\dot{\lambda}}\left(\frac{\partial j_{\dot{m}}}{\partial \psi_{\dot{\lambda}}} \frac{\partial j_{\dot{ }}}{\partial \chi^{\dot{\lambda}}}-\frac{\partial j_{\dot{m} k}}{\partial \chi_{\dot{\lambda}}} \frac{\partial j_{\dot{m} l}}{\partial \psi_{\dot{\lambda}}}\right) .
\end{aligned}
$$

Dabei entsteht offenbar wieder ein bilinearer Ausdruck; allgemein führen $\mathrm{P}$ ois s on-Klammern $\mathrm{zwischen} \mathrm{bilinearen} \mathrm{Größen} \mathrm{wieder} \mathrm{auf} \mathrm{bilineare.}$ Alle bilinearen Spinorkombinationen, die- so entstehen, entsprechen aber Vektoren oder Tensoren - es ist eine Besonderheit der Spinorrechnung, $\mathrm{da} ß$ auch die Invarianten und antisymmetrischen Tensoren von gleicher Faktorenstufe wie die Vektoren sind -, man bleibt also mit der Bildung der Poisson-Klammern beständig innerhalb der Gruppe der Welttensoren. Die Spinoren fallen ganz dabei heraus; die Zurückführbarkeit der Tensor- auf Spinorrelationen bedeutet nur eine Einschränkung in den Möglichkeiten der ersteren.

Eine solche Einschränkung ist erwünscht, denn ein System von Vertauschungsrelationen zwischen Tensorkomponenten ist noch weitgehend frei wählbar und keineswegs allein durch die relativistische Invarianz festgelegt. Für Spinoren 1. Stufe lassen sich aber nur zwei invariante Systeme von Poisson-Klammern bilden, und nur mit dem System (2.4) bleibt man innerhalb der j, und $M_{i k}$. Wir zeigen zunächst die Invarianz. Seien $\psi^{\prime}, \chi^{\prime}$ die Spinoren in einem bewegten System, so drückt sich eine Lorentz-Transformation auf das ruhende bekanntlich durch eine binäre Transformation

$$
\psi_{k}^{\prime}=a_{k \cdot \mu} \psi_{\mu} \quad \%_{k}^{\prime}=a_{k, \mu} \psi_{\mu} \quad k, \mu=1,2
$$

mit der Koeffizientendeterminante $|\alpha|=1$ aus. Dazu treten immer die komplex konjugierten Formeln. Es ist also (beachte $\psi^{1}=\psi_{2}, \psi^{2}=-\psi_{1}$ ):

$$
\begin{aligned}
& \left(\psi_{1}^{\prime} \chi^{1 \prime}\right)=\left({\psi_{1}}^{\prime} \chi_{2}{ }^{\prime}\right) \\
& =\left(a_{11} \psi_{1}+a_{12} \psi_{2}, a_{21} \chi_{1}+a_{22} \chi_{2}\right) \\
& =a_{11} \alpha_{21}\left(\psi_{1} \chi_{1}\right)+\alpha_{11} a_{22}\left(\psi_{1} \chi_{2}\right) \\
& +\alpha_{12} a_{21}\left(\psi_{2} \chi_{1}\right)+\alpha_{12} a_{22}\left(\psi_{2} \chi_{2}\right) \\
& =-a_{11} a_{21}\left(\psi_{1} \chi^{2}\right)+\alpha_{11} a_{22}\left(\psi_{1} \chi^{1}\right) \\
& -\alpha_{12} \alpha_{21}\left(\psi_{2} \chi^{2}\right)+a_{12} \alpha_{22}\left(\psi_{2} \chi^{1}\right) \\
& =\left(\alpha_{11} \alpha_{22}-\alpha_{12} \alpha_{21}\right) \cdot 1=1 \\
& \left(\psi_{1}{ }^{\prime} \chi^{2 \prime}\right)=-\left(\psi_{1}{ }^{\prime} \chi_{1}{ }^{\prime}\right) \\
& =-\left(\alpha_{11} \psi_{1}+\alpha_{12} \psi_{2}, \alpha_{11} \chi_{1}+\alpha_{12} \chi_{2}\right) \\
& =-a_{11}^{2}\left(\psi_{1} \chi_{1}\right) \cdot-\alpha_{11} \alpha_{12}\left(\psi_{1} \chi_{2}\right) \\
& -a_{12} a_{11}\left(\psi_{2} \chi_{1}\right)-\alpha_{12}^{2}\left(\psi_{2} \psi_{2}\right) \\
& =a_{11}^{2}\left(\psi_{1} \chi^{2}\right)-\alpha_{11} a_{12}\left(\psi_{1} \chi^{1}\right) \\
& +\alpha_{12} a_{11}\left(\psi_{2} \chi^{2}\right)-\alpha_{12}^{2}\left(\psi_{2} \chi^{1}\right)=0
\end{aligned}
$$

usw., was zu beweisen war. Man sieht sofort, daß kanonische Konjugation komplex konjugierter Spinoren, etwa in der Form $\left(\psi_{1} \psi^{1}\right)$, nicht in Frage kommt, denn die Transformationskoeffizienten konjugiert-komplexer $\psi$ sind komplex konjugiert zu denen der $\psi$ und bilden damit nicht die Determinante 1. Dagegen wäre es relativistisch noch möglich, $\left(\psi_{\mu} \psi^{v}\right)=\left(\chi^{\mu} \chi^{v}\right)=\delta_{\mu}^{v}$ zu verlangen und alle $(\psi \chi)$ verschwinden $\mathrm{zu}$ lassen. Die hiermit zu bildenden $\mathrm{P}$ o is s on-Klammern führen aber, wie wir im 4. Abschnitt an einem Beispiel zeigen werden, aus dem System der $j$ - und $M$-Komponenten heraus. Die Formeln (2.4) sind also eindeutig ausgezeichnet, und wir wollen das in Anbetracht ihrer besonderen mathematischen Einfachheit zum Anlaß nehmen, die Theorie hierauf aufzubauen. Dieses Prinzip ist natürlich rein heuristisch; entscheidend ist, ob man damit bei Wahl einer geeigneten Hamilton-Funktion wirklich in erster Näherung die Bewegungsgleichung mit der Strahlungskraft für ein punktförmiges Teilchen wiederfindet.

\section{Systematik der Tensoren}

Hier wollen wir nun alle ins Spiel tretenden Größen mit ihren gegenseitigen Beziehungen in Spinor-, Tensor- und Vektorform zusammenstellen. Wir folgen dabei durchaus L a p or te und Uhlenbeck, denen wir uns auch in den Bezeichnungen 
anschließen. Die Vierervektoren $j$ und $k$ werden also in Spinor- und Tensorform gleich geschrieben, da sie sich durch die Anzahl der Indices unterscheiden; den Momententensor schreiben wir dagegen als Spinor mit kleinen, als Tensor mit großen Buchstaben, weil er in beiden Fällen zweifach indiziert ist. Auch in der Metrik wollen wir Laporte und Uhlenbeck folgen und die Koeffizienten des Weltlinienelements $g_{11}=g_{22}$ $=g_{33}=1, g_{44}=-1$ setzen. Wir verlassen damit die in unsern früheren Arbeiten benutzte „pseudoeuklidische“ Metrik, denn da wir schon mit komplexen Spinoren rechnen und später Vertauschungsrelationen einzuführen beabsichtigen, erscheint es wünschenswert, einen weiteren Gebrauch der imaginären Einheit zu vermeiden. Summationen iiber doppelte Indices gehen bei Spinoren von 1 bis 2, bei Tensoren von 1 bis 4 .

Aus den vier komplexen Spinoren $\psi_{1}, \psi_{2}, \chi_{1}, \chi_{2}$ und ihren komplex Konjugierten können wir nun bilden

I. die zwei Invarianten

$$
\Delta=\psi_{\lambda} \chi^{\lambda} \quad \Delta=\psi_{\dot{\lambda}} \chi^{\dot{\lambda}} .
$$

Ihre Real- und Imaginärteile heißen

$$
I=\frac{1}{2}(\Delta+\bar{\Delta}) \quad J=\frac{1}{2 i}(\Delta-\Delta) .
$$

Die Invarianten sind auch die einzige Kennzahl des antisymmetrischen Spinors zweiter Stufe:

$$
\begin{aligned}
& \psi_{r} \chi^{s}-\chi_{r} \psi^{s}=\delta_{r}^{s} \Delta \\
& \psi_{r} \chi^{\dot{s}}-\chi_{r} \psi^{\dot{s}}=\delta_{r}^{\dot{s}} \bar{\Delta}
\end{aligned}
$$

II. Die beiden Vierervektoren

$$
\begin{aligned}
& j_{\dot{m} l}=\psi_{\dot{m}} \psi_{l}+\chi_{\dot{m}} \chi_{l} \\
& k_{\dot{m} l}=\psi_{\dot{m}} \psi_{l}-\chi_{\dot{m}} \chi_{l} .
\end{aligned}
$$

Sie stehen aufeinander senkrecht:

$$
k_{\sigma \lambda} \dot{j}^{\dot{\sigma} \lambda}=0
$$

und ihre Quadrate drücken sich durch die $\triangle$ aus (beachte $\psi \lambda \psi^{\lambda}=0$ !) :

$$
j_{\sigma \lambda} j^{\dot{\sigma} \lambda}=2 \Delta \bar{\Delta}=-k_{\sigma \lambda} l_{i}^{\dot{\sigma} \lambda}
$$

Die beiden noch möglichen Kombinationen $\psi$ in $\chi_{l} \pm \chi$ in $\psi_{l}$ bilden übrigens ein untereinander und $\mathrm{zu} j$ und $k$ orthogonales Vektorenpaar. Man kann so aus den vier Spinoren gerade ein orthogonales Vierbein herstellen. - Endlich haben wir

III. den Momententensor

$$
\begin{gathered}
m_{\ddot{r} s}=\frac{\Lambda}{i}\left(\psi_{r} \chi_{s}+\psi_{s} \chi_{\dot{r}}\right) \\
m_{r s}=-\frac{A}{i}\left(\psi_{r} \chi_{s}+\psi_{s} \chi_{r}\right) .
\end{gathered}
$$

Die bei La p or te und U h.le $\mathrm{n}$ be $\mathrm{ck}$ als Faktor stehende Länge $h / m c$ (C o m p t o n - Wellenlänge) ist hier durch den Buchstaben $\Lambda$ ersetzt. Die Invarianten des Tensors sind

$$
\begin{aligned}
& m_{\varrho \sigma} m^{\varrho \sigma}=2 \Lambda^{2} \Delta^{2} \\
& m_{\varrho \sigma} \dot{\sigma} m^{\dot{\sigma} \sigma}=2 \Lambda^{2} \bar{\Delta}^{2},
\end{aligned}
$$

und seine Verjüngung mit $j$ liefert $k$ und umgekehrt:

$$
\begin{aligned}
& \dot{j}_{l} m_{\varrho s}=-\frac{A}{i} k_{s l} \bar{\Delta} \\
& k_{\varrho_{l}} m_{\varrho_{s}} \ddot{\Delta}=-\frac{A}{i} j_{\dot{s} l} \Delta .
\end{aligned}
$$

An dieser Stelle wollen wir uns auch über die Dimension unserer neuen Variablen Rechenschaft geben. Aus (3.7) folgt, da die Momente die Dimension Ladung mal Länge haben, für die Produkte der $\psi, \chi$ die Dimension Ladung. Die Invarianten $I$ und $J$ haben also die Dimension einer Ladung. Zufolge (3.4) müssen die $\psi$ und $\chi$ dimensionsgleich sein. Die Vektoren erscheinen also auch unter der Dimension Ladung. Wir können auch hieraus schließen, wie wir das schon in $\mathrm{Ab}$ schnitt 1 aus der Abzählung der unabhängigen Veränderlichen gefolgert haben, daß im Rahmen dieser Betrachtungen - anders als in der Wellenmechanik - nur die aus ihnen gebildeten Vierergeschwindigkeiten von Bedeutung sind.

Wir wollen nun die algebraischen Beziehungen in Tensorschreibweise wiederholen. Zur Übersetzung dient das bekannte Schema der Vektoren

$$
\begin{aligned}
& j^{1}=\frac{1}{2}\left(j_{21}+j_{12}\right)=j_{1} \quad j^{3}=\frac{1}{2}\left(j_{11}-j_{22}\right)=j_{3} \\
& j^{2}=\frac{1}{2 i}\left(j_{21}-j_{12}\right)=j_{2} \quad j^{4}=\frac{1}{2}\left(j_{11}+j_{22}\right)=-j_{4}
\end{aligned}
$$

nebst dem folgenden, bei $L$ a p o r te und U h l e n beck nicht explicite angegebenen der antisym- 
metrischen Tensoren (der Stern deutet den dualen Tensor an):

$$
\begin{aligned}
& M_{12}=-\frac{1}{2 i}\left(m_{12}-m_{\ddot{12}}\right)=i M_{43}^{\ddot{*}} \\
& M_{23}=\frac{1}{4 i}\left(m_{11}-m_{\ddot{11}}-m_{22}+m_{\ddot{2} 2}\right)=i M_{41}^{*} \\
& M_{31}=-\frac{1}{4}\left(m_{11}+m_{\ddot{11}}+m_{22}+m_{\ddot{22}}\right)=i M_{42}^{*} \\
& M_{41}=\frac{1}{4}\left(m_{11}+m_{\ddot{11}}-m_{22}-m_{\ddot{2} 2}\right)=-i M_{23}^{*} \\
& M_{42}=\frac{1}{4 i}\left(m_{11}-m_{\ddot{11}}+m_{22}-m_{2 \ddot{2}}\right)=-i M_{31}^{*} \\
& M_{43}=-\frac{1}{2}\left(m_{12}+m_{\ddot{12}}\right)=-i M_{12}^{*} .
\end{aligned}
$$

Hiermit wollen wir zunächst die beiden Invarianten $M_{i k} M^{i k}$ und $M_{i k}^{*} M^{i k}$ von $M$ durch $I$ und $J$ ausdrücken. Durch Ausmultiplizieren nach (3.11) ergibt sich mit Rücksicht auf (3.8) und (3.2):

$$
\frac{1}{2} M_{i k} M^{i *}=A^{2}\left(I^{2}-J^{2}\right) \quad i M_{i k}^{*} M^{i k}=4 A^{2} I J .
$$

Aus (3.6) wird nach (3.10) und (3.2):

$$
-j_{r} j^{r}=I^{2}+J^{2}=k_{r} k^{r}
$$

und die Beziehungen (3.9) bedeuten

$$
\begin{aligned}
& j^{r} M_{r s}=\Lambda J k_{s} \\
& k^{r} M_{r s}=\Lambda J j_{s},
\end{aligned}
$$

was man durch Multiplikation mit $M^{* s t}$ unter Beachtung von

$M_{r s} M^{* s t}=M_{r s}^{*} M^{s t}=-\frac{1}{4} \delta_{r}^{t}\left(M^{*} M\right)=i \delta_{r}^{t} A^{2} I . J$ auch in

$$
\begin{aligned}
& k_{r} M_{r s}^{*}=i \Lambda I j_{s} \\
& j^{r} M_{r s}^{*}=i \Lambda I k_{s}
\end{aligned}
$$

verwandeln kann. Damit haben wir die sieben Bedingungen, von denen in Abschnitt 1 die Rede war, zusammengestellt: durch (3.12) werden die Invarianten $I$ und $J$, durch die erste Gl. (3.13) die von $j$ an die des Momententensors angeschlossen, und (3.14) oder (3.15) drücken $k$ durch $j$ und $M$ aus.

Wir führen nun gemäß Abschnitt 1 die beiden
Vierervektoren $u$ und $U$ ein und gehen zu Raumvektoren über. Zur Abkürzung werde

$$
I^{2}+J^{2}=l^{2}
$$

gesetzt, so daß $l=|j|$ ist. Dann ist

$$
u^{i}=j^{i} / l
$$

mit den Komponenten

$\mathfrak{u}=\frac{\mathfrak{v} / c}{\sqrt{1-v^{2} / c^{2}}} \quad u^{4}=\frac{1}{\sqrt{1-v^{2} / c^{2}}}=-u_{4}$

und

$$
U^{i}=k^{i} / l
$$

mit den Komponenten

$\mathfrak{U}=\frac{\mathfrak{B} / c}{\sqrt{V^{2} / c^{2}-1}} U^{4}=\frac{1}{\sqrt{V^{2} / c^{2}-1}}=-U_{4}$.

Es gilt

$$
\begin{gathered}
u_{j} u^{j}=\mathfrak{v}^{2}-u_{4}^{2}=-1 \\
U_{j} C^{j}=\mathfrak{U}^{2}-U_{4}^{2}=+1
\end{gathered}
$$

und wegen (3.5)

$$
u_{j} U^{j}=O
$$

oder $\mathfrak{b} \mathfrak{B} / c^{2}-1=0$, worauf schon in Abschnitt 1 hingewiesen wurde.

Endlich zerlegen wir den Momententensor in das magnetische und elektrische Moment $\mathfrak{M}$ und $\mathfrak{P}$ gemäß ${ }^{14}$

$$
\left(M_{23}, M_{31}, M_{12},\right)=\mathfrak{M} \quad\left(M_{14}, M_{24}, M_{34},\right)=\mathfrak{B} .
$$

Dann schreiben sich die Gl. (3.12)

$\mathfrak{M}^{2}-\mathfrak{B}^{2}=\Lambda^{2}\left(I^{2}-J^{2}\right) \quad \mathfrak{B} \mathfrak{M}=-\Lambda^{2} I J,(3.24)$

und die ersten Gl. (3.14), (3.15) gehen nach Division durch $l$ mit Benutzung von (3.17), (3.18) und Elimination der jeweils vierten über in

$$
\frac{\mathfrak{B}}{c}=\frac{\mathfrak{P}+[\mathfrak{v} \mathfrak{M}] / \mathrm{c}}{\mathfrak{v} \mathfrak{P} / \mathrm{c}}=\frac{\mathfrak{M}-[\mathfrak{v} \mathfrak{P}] / \mathrm{c}}{\mathfrak{v} \mathfrak{M} / \mathrm{c}} .
$$

Auf diese Weise drückt sich also die zugeordnete Geschwindigkeit durch die gewöhnliche aus. Zur Umkehrung der Formeln braucht man bloß

${ }^{14}$ In den beiden vorangehenden Arbeiten haben wir den Buchstaben $\mathfrak{F}$ zur Abkürzung von $\mathfrak{p}+\frac{e}{c} \mathfrak{A}$ verwendet. In der hier gebrauchten Bedeutung ist er aber so geläufig, daß wir lieber die Abkürzung abändern wollen. 
$\mathfrak{B}$ und $\mathfrak{v} \mathrm{zu}$ vertauschen, wie aus (3.14), (3.15) unmittelbar $\mathrm{zu}$ ersehen ist.

Die Größen $\mathfrak{M}, \mathfrak{P}$ und $\mathfrak{v}$ oder auch $M_{i k}$ und $u^{j}$ sind nun also unsere neun Variablen neben den Koordinaten und Impulsen. Es bleibt noch anzumerken, daß sie noch einer invarianten Bindung unterliegen. Wir haben ja alles aus vier komplexen Spinoren aufgebaut; es können also nur acht Größen wirklich unabhängig sein. Die Invarianten von $j$ und $M$ wurden schon berücksichtigt; es bleibt aber noch die von $j M$ übrig, das ist das Quadrat von (3.14), und dies führt in der Tat wegen der zweiten Hälfte der Gleichung (3.13) auf $j^{2}$ zurück. In Raumvektoren lautet die Beziehung

$$
(\mathfrak{P}+[\mathfrak{v} \mathfrak{M}] / \mathfrak{c})^{2}-(\mathfrak{B} \mathfrak{v} / \mathfrak{c})^{2}=A^{\prime} J^{2}\left(1-\frac{v^{2}}{c^{2}}\right) .
$$

Dies wird also immer ein partikuläres Integral unserer Bewegungsgleichungen sein. Weitere Invarianten lassen sich aus $j$ und $M$ nicht bilden, denn Produkte von mehr als zwei gleichen antisymmetrischen Tensoren lassen sich immer auf Ausdrücke mit einem oder zwei Faktoren zurückführen ${ }^{15}$.

\section{Die Pois on - Klammern}

Nach diesen Vorbereitungen können wir zur Bildung der Poisson-Klammern übergehen. Den Ansatz dazu können wir noch ein wenig erweitern, indem wir annehmen, daß die Formeln (2.4) bzw. (2.5) nur für gewisse ursprüngliche Spinoren $\psi_{0}, \chi_{0}$ gelten, von denen sich die $\psi, \chi$ noch um einen Faktor unterscheiden. Es sei also

$$
\begin{aligned}
& \left(\psi_{\mu} \chi^{\nu}\right)=\delta_{\mu}{ }^{v} I \mathrm{e}^{i \delta} \\
& \left(\psi_{\dot{\mu}} \chi^{\dot{\nu}}\right)=\delta_{\dot{\mu}} \dot{\nu} T \mathrm{e}^{-i \delta}
\end{aligned}
$$

und alle übrigen Klammern Null. Wir haben den
Faktor komplex angesetzt, weil die Spinoren komplex sind, und er wird sich sogar als rein imaginär herausstellen. Das hat nichts mit dem Auftreten der imaginären Einheit in den Vertauschungsrelationen $\mathrm{zu}$ tun. Wir treiben hier klassische Theorie, und die Poisson-Klammern der reellen Tensoren müssen demgemäß durchweg reell werden. Das ist auch der Fall, denn in Formel (2.5), in der man sich jetzt die $\psi_{0}, \chi_{0}$ an Stelle der $\psi, \chi$ zu denken hat, gehen ja diese und ihre komplex Konjugierten symmetrisch ein.

Mit dem Übergange von (2.4) $\mathrm{zu}$ (4.1) nehmen die beiden Summen in (2.5) den Faktor $\Gamma e^{i \delta} \mathrm{bzw}$. $\Gamma e^{-i \delta}$ an. Solange wir es nur mit den bilinearen Ausdrücken zu tun haben, können wir diese Formel auch ganz beiseite lassen und mit (4.1) wie mit Vertauschungsrelationen rechnen. Wir werden auch Größen, deren P o is s on-Klammern verschwinden, wie in der Quantenmechanik als vertauschbar bezeichnen. - Bei den folgenden Umformungen sind die leicht $\mathrm{zu}$ beweisenden Regeln

$$
\begin{aligned}
& \left(\gamma_{\mu} \psi^{\nu}\right)=\left(\psi_{\mu} \chi^{v}\right) \\
& \left(\chi_{\mu} \psi^{\dot{v}}\right)=\left(\psi_{\mu} \chi^{\nu^{v}}\right)
\end{aligned}
$$

und die bekannte Formel $\psi_{\lambda} \chi^{\lambda}=-\psi_{\lambda} \chi^{\lambda}$ sowie die daraus folgende $\psi_{\lambda} \psi^{\lambda}=0$ mit ihren Konjugierten häufig anzuwenden.

Vertauschbar sind offenbar die $\Delta$ und $\Delta$ von (3.1), weil die $\psi, \chi$ und ihre komplex Konjugierten es sind; daher sind auch die Invarianten $I$ und $J$ vertauschbar. Die Berechnung der übrigen Kombinationen vereinfacht sich sehr, wenn man mit denen der Momente untereinander beginnt, weil sie $\mathrm{zu}$ einer bestimmten, vereinfachenden Wahl der Phase $\delta$ in (4.1) Anlaß geben. Wir bilden also

$$
\begin{aligned}
\left(m_{\mu \nu} m^{o \sigma}\right) & =-\Lambda^{2}\left(\psi_{\mu} \chi_{\nu}+\chi_{\mu} \psi_{\nu}, \psi^{\varrho} \chi^{\sigma}+\chi^{o} \psi^{\sigma}\right) \\
& =-\Lambda^{2} T \mathrm{e}^{i \delta} \psi_{\mu} \delta_{\nu}{ }^{o} \chi^{\sigma}+\chi_{\nu} \delta_{\mu}{ }^{\sigma} \psi^{\varrho}+\text { usw. } \\
& =i \Lambda T \mathrm{e}^{i \delta}\left\{\delta_{\nu}{ }^{o} m_{\mu}{ }^{\sigma}+\delta_{\nu}{ }^{\sigma} m_{\mu}{ }^{o}+\delta_{\mu}{ }^{o} m_{\nu}{ }^{\sigma}+\delta_{\mu}{ }^{\sigma} m_{\nu}{ }^{\varrho}\right\}
\end{aligned}
$$

nebst dem konjugierten Ausdruck, der dadurch entsteht, daß man die Indices punktiert und $i$ in $\boldsymbol{- i}$ verwandelt. Nun ist nach (3.11) z. B.

15 W. W e s s e l, Z. Physik 110, 625 [1938], Schlußabschnitt. 
Zufolge (4.3) ist aber

$$
\begin{aligned}
\left(M_{23} M_{31}\right) & =-\frac{1}{16 i}\left(m_{11}-m_{11}-m_{22}+m_{22}, m_{11}+m_{1 i}+m_{22}+m_{22}\right) \\
& =-\frac{1}{8 i}\left\{\left(m_{11} m_{22}\right)-\left(m_{11} m_{22}\right)\right\} \\
& =-\frac{1}{8 i}\left\{\left(m_{11} m^{11}\right)-\left(m_{1 i} m^{i 1}\right)\right\}
\end{aligned}
$$

und folglich

$$
\begin{aligned}
& \left(m_{11} m^{11}\right)=4 i \Gamma \Lambda e^{i \delta} m_{1}{ }^{1}=4 i \Lambda I e^{i \delta} m_{12} \\
& \left(m_{1 \mathrm{i}} m^{1 \mathrm{i}}\right)=-4 i \Lambda \Gamma e^{-i \delta} m_{1} \cdot \dot{1}=-4 i \Lambda T e^{-i \delta} m_{12}
\end{aligned}
$$

$$
\begin{aligned}
\left(M_{23} M_{31}\right) & =-\Lambda T\left\{\frac{1}{2}\left(m_{12}+m_{12}\right) \cos \delta+\frac{i}{2}\left(m_{12}-m_{12}\right) \sin \delta\right\} \\
& =-\Lambda T\left\{M_{12} \sin \delta+i M_{12}^{*} \cos \delta\right\} .
\end{aligned}
$$

Diese P o iss o n-Klammer für das magnetische Moment mit ihren zyklisch zu ergänzendeli entspricht gerade der für einen Drehimpuls $d_{i k}$ geltenden

$$
\left(d_{23} d_{31}\right)=-d_{12} \text { usw. }
$$

wenn man $\cos \delta=0$ setzt. Da man sich bei einem negativen Elektron das magnetische Moment dem Eigendrehimpuls entgegengesetzt gerichtet vorzustellen hat, werden wir es so einrichten, daß

$$
\left(M_{23} M_{31}\right)=+\Gamma \Lambda M_{12}
$$

wird. Demgemäß haben wir

$$
\delta=-\pi / 2
$$

zu setzen. Die Fundamentalrelationen (4.1) gehen damit über in

$$
\begin{aligned}
& \left(\psi_{\mu} \chi^{v}\right)=\frac{\Gamma}{i} \delta_{\mu}{ }^{v} \\
& \left(\psi_{\mu} \chi^{v}\right)=-\frac{I}{i} \delta_{\mu}{ }^{v} .
\end{aligned}
$$

Aus einem Vergleich von (4.7) und (4.8) können wir auf die Dimension von $\Gamma$ schließen, wenn wir über die noch unbestimmt gelassene unserer $\Psi_{0}, \chi_{0}$ so verfügen, daß sich die Bewegungsgleichungen alle einheitlich in der gewohnten Form durch Bildung der Poisson-Klammern der betreffenden Veränderlichen mit der Hamil to n schen Funktion ableiten lassen. Dann nimmt $\Lambda \Gamma$ die Dimension magnetisches Moment: Drehimpuls an, und da $\Lambda$ die einer Länge, das Moment die
Dimension Ladung mal Länge und der Drehimpuls die einer Wirkung hat, folgt für $\Gamma$ :

$$
\left[I^{\prime}\right]=\text { Ladung/Wirkung }
$$

Man kann auch schließen: Die Dimension des Produktes der $\psi_{0}, \chi_{0}$, nach denen in (4.10) differentiiert $\mathrm{zu}$ denken ist, muß auf Grund der eben erhobenen Forderung die eines Produktes von Impulsen und Koordinaten, also einer Wirkung, sein, und da die $\psi, \chi$-Produkte nach dem früher Gesagten Ladungen entsprechen, folgt aus (4.10) für $\Gamma$ wieder die Dimension Ladung: Wirkung.

Hiernach gehen wir zum systematischen Aufbau sämtlicher möglichen $\mathrm{P}$ o i s s o n-Klammern über. Die Vertauschbarkeit der Invarianten I und $J$ wurde schon festgestellt. Es folgt die Kombination Invarianten und Vektoren. Wir haben z. B.

$$
\begin{aligned}
\left(j_{\dot{m} n} \dot{\Delta}\right) & =\left(\psi_{\dot{m}} \psi_{n}+\chi_{\dot{m}} \chi_{n}, \psi_{\lambda} \chi^{\lambda}\right) \\
& =\left(\psi_{\dot{m}} \psi_{n}, \psi_{\lambda} \chi^{\lambda}\right)-\left(\chi_{\dot{m}} \chi_{n}, \psi^{\lambda} \chi_{\lambda}\right) \\
& =\frac{\Gamma}{i}\left\{\psi_{\dot{m}} \psi_{n}-\chi_{\dot{m}} \chi_{n}\right\}=\frac{I}{i} k_{\dot{m} n}
\end{aligned}
$$

oder, da man hier offenbar gleich von den Spinoren auf die Vektoren schließen kann:

$$
\left(j^{l} \Delta\right)=\frac{\Gamma}{i} k^{l}
$$

usw. und schließlich

$$
\begin{array}{ll}
\left(j^{l} I\right)=0 & \left(j^{l} J\right)=-T k^{l} \\
\left(k^{l} I\right)=0 & \left(k^{l} J\right)=-I j^{l} .
\end{array}
$$


$\boldsymbol{I}$ ist also auch mit den Vektoren vertauschbar. An dieser Stelle sei angemerkt, daß man mit den am Schlusse von Abschnitt 2 erwähnten $\mathrm{P}$ o is s o n-Klammern erhalten würde

$$
\left(j_{m n} \Delta\right)=-\psi_{m} \chi_{n}+\chi_{\dot{m}} \psi_{n}
$$

das ist einer der im Anschluß an (3.6) genannten, auf $j$ und $k$ senkrechten Vektoren, denen keine bekannte Bedeutung zukommt:

Die Velitoren untereinander bilden

$$
\begin{aligned}
&\left(k_{\dot{m} n} \dot{j}^{\dot{r}}\right)=\frac{I}{i}\left\{\delta_{n}^{s}\left(\psi_{\dot{m}} \chi^{\dot{r}}-\chi_{\dot{m}} \psi^{\dot{r}}\right)\right. \\
&\left.\quad-\delta_{\dot{m}}\left(\psi_{n} \chi^{s}-\chi_{n} \psi^{s}\right)\right\} .
\end{aligned}
$$

Hier treten die unter (3.3) erwähnten antisymmetrischen Spinoren auf; wir haben also

$\left(k_{m n} \dot{j}^{\dot{s}}\right)=\frac{\Gamma}{i} \delta_{n}{ }^{s} \delta_{m} \dot{r}(\bar{\Delta}-\Delta)=-2 \Gamma J \delta_{n}{ }^{s} \delta_{\dot{m}} \dot{r}$.

Bei der Umrechnung auf die $j, k$ hebt sich der Faktor -2, und es wird

$$
\left(k_{r} j^{s}\right)=\delta_{r}^{s} \Gamma J .
$$

Die Kombination der Vektorkomponenten untereinander verläuft ganz ähnlich und ergibt

$$
\left(k_{i} k_{k}\right)=\frac{T}{A} M_{i k}=-\left(j_{i} j_{k}\right) \text {. }
$$

Unser ursprünglich nur unter dem Gesichtspunkte mathematischer Einfachheit gewählter Ansatz bringt uns hier wieder in befriedigenden Einklang mit der D i r a c schen Theorie, in der, nach Laporte und Uhlenbeck, die räumlichen Komponenten der $k_{i}$ dem mechanischen Drehimpuls des Elektrons entsprechen: zwar ist nicht, weil aus Transformationsgründen nicht möglich, $\left(k_{1}, k_{2}\right) \sim k_{3}$, wohl aber

$$
\left(k_{1} k_{2}\right)=\frac{I}{\Lambda} \mathfrak{M}_{3},
$$

d.h. der Komponente des magnetischen Moments in der Richtung von $k_{3}$ proportional.

Die noch ausstehenden Kombinationen der Momente mit den übrigen Größen können wir nun unter Vंermeidung der Spinorrechnung sehr einfach bilden, indem wir nach (4.17) die Momente durch die $\left(k k_{k}\right)$ oder $\left(j_{i} j_{k}\right)$ ausdrücken. Die Momente und Invarianten bilden so: erstens, da $I$ mit sämtlichen Vektoren vertauschbar ist,

$$
\left(I M_{i h}\right)=0
$$

und zweitens, unter Anwendung $\operatorname{der}_{\mathrm{s}} \mathrm{J}$ a k o bi . schen Identität,

$$
\begin{aligned}
&\left(J M_{i k}\right)=\frac{\Lambda}{T}\left(J\left(k_{i} k_{k}\right)\right) \\
&=-\frac{\Lambda}{\Gamma}\left\{\left(k_{k}\left(J k_{i}\right)\right)+\left(k_{i}\left(k_{k} J\right)\right)\right\} \\
&=\Lambda\left\{\left(k_{k} j_{i}\right)-\left(k_{i} j_{h}\right)\right\} \\
&=-\Lambda \Gamma J\left(g_{k i}-g_{i k}\right)=0 .
\end{aligned}
$$

Die Momente und Invarianten sind also durchweg vertauschbar. Die Invariante I ist überhaupt mit allen Größen vertauschbar und folglich eine Konstante der Bewegung.

Bei der Kombination der Momente und Vektoren drücken wir $M_{i k}$ gegenüber $j$ durch $\left(k_{i} k_{k}\right)$, gegenüber $k$ durch $\left(j_{i} j_{k}\right)$ aus und finden so, wieder unter Anwendung der J akobi schen Identität,

$$
\begin{aligned}
& \left(M_{2} k^{l}\right)=\Lambda T\left\{\delta_{i}^{l} k_{k}-\delta_{k}^{l} k_{i}\right\} \\
& \left(M_{i k} j^{l}\right)=\Lambda T\left\{\delta_{i}^{\prime} j_{k}-\delta_{k}^{l} j_{i}\right\} .
\end{aligned}
$$

Schließlich ergibt sich ganz ähnlich, unter Heranziehung der eben gewonnenen Formeln, für die Momentkomponenten:

$$
\begin{array}{r}
\left(M_{i k} M^{r s}\right)=\Lambda I\left\{\delta_{i}^{r} M_{k}{ }^{s}-\delta_{k}{ }^{r} M_{i}^{s}-\delta_{i}^{s} M_{k}^{r}\right. \\
+\delta_{k}^{s} M_{i}^{r}
\end{array}
$$

wovon (4.8) bereits ein spezieller Fall war.

Alle diese Veränderlichen sollen zu den Lagenkoordinaten und Impulsen hinzutreten und mit ihnen uneingeschränkt vertauschbar sein. Die Formel (2.5) ist dementsprechend noch durch eine Summe ergänzt zu denken, in der nach den $p_{k}, q^{k}$ differentiiert wird.

5. Die Poisson-Klammern der $u_{i}, \quad U$ und $M_{i k}$

Die Vierervektoren $j$ und $k$ sind, wie mehrfach betont wurde, im Rahmen unserer Betrachtungen nur Hilfsgrößen, von denen bloß die „Einheitsvektoren" $u_{i}=j_{\ell} / j /|j|$ und $U_{t}=k_{i} /|k|$ Bedeutung haben. Wir kommen nun zu den endgültigen Beziehungen. $\mathrm{Zu}$ ihnen gehören aus dem Vorángehenden schon die von den $j$ und $k$ freien $\mathrm{P}$ o i s so n-Klammern (4.22) der Momente unterein- 
ander, die wir daher hier, in $\mathfrak{M}$ und $\mathfrak{P}$ geschrieben, noch einmal zusammenstellen:

$$
\begin{gathered}
\left(\mathfrak{M}_{1} \mathfrak{M}_{2}\right)=I \Lambda \mathfrak{M}_{3}=--\left(\mathfrak{P}_{1} \mathfrak{P}_{2}\right) \\
\left(\mathfrak{M}_{1} \mathfrak{P}_{2}\right)=I \Lambda \mathfrak{P}_{3} \\
\left(\mathfrak{M}_{1} \mathfrak{P}_{1}\right)=\left(\mathfrak{M}_{2} \mathfrak{P}_{2}\right)=\left(\mathfrak{M}_{3} \mathfrak{P}_{3}\right)=0 .
\end{gathered}
$$

Die ersten drei sind durch zyklische Vertauschung der Indices zu ergänzen. Durch einfache Division mit $l$ sind auch die Beziehungen (3.14) und (3.15) ohne weiteres auf die $u_{i}, U_{i}$ umzurechnen:

$$
\begin{array}{ll}
u^{r} M_{r s}=\Lambda J U_{s} & u^{r} M_{r s}^{*}=i \Lambda J U_{s} \\
U^{r} M_{r s}=\Lambda J u_{s} & U^{r} M_{r s}^{*}=i \Lambda J u_{s} .
\end{array}
$$

Um die P o is s o n-Klammern der $u_{i}, U_{i}$ zu bilden, müssen wir jetzt auf die (mit den Faktoren $\Gamma / i$ und $-\Gamma / i$ ergänzte) Differentiationsformel (2.5) zurückgreifen. Danach ist, wenn $X$ irgendeine der vorkommenden Größen bedeutet, mit Rücksicht auf die Definition (3.16) von $l$ :

$$
\begin{aligned}
\left(\frac{1}{l} X\right) & =(I X) \frac{\partial}{\partial I}\left(\frac{1}{l}\right)+(J X) \frac{\partial}{\partial J}\left(\frac{1}{l}\right) . \\
& =-\frac{J}{l^{3}}(J X),
\end{aligned}
$$

da I mit allen Größen vertauschbar ist. Es folgt

$$
\begin{aligned}
\left(u_{i} X\right) & =\left(\frac{j_{i}}{l} X\right)=\frac{1}{l}\left(j_{i} X\right)+j_{i}\left(\frac{1}{l} X\right) \\
& =\frac{1}{l}\left(j_{i} X\right)-\frac{J}{l^{3}} j_{i}(J X)
\end{aligned}
$$

oder

$$
\begin{aligned}
\left(u_{i} X\right) & =\frac{1}{l}\left(i_{j} X\right)-\frac{J}{l^{2}} u_{i}(J X) \\
\left(U_{i} X\right) & =\frac{1}{l}\left(k_{i} X\right)-\frac{J}{l^{2}} U_{i}(J X) .
\end{aligned}
$$

Für $X=J$ ist z. B. wegen (4.13)

$$
\begin{aligned}
& \left(u_{i} J\right)=-I U_{i} \\
& \left(U_{i} J\right)=-I u_{i} .
\end{aligned}
$$

Weiter hat man die $\left(u_{i} j_{k}\right),\left(u_{i} k_{k}\right)$ usw. zu bilden, um dann zu den $\left(u_{i} u_{k}\right),\left(u_{i} U_{k}\right)$ und $\left(U_{i} U_{k}\right)$ überzugehen. Wir geben gleich die Endformeln:

$$
\begin{aligned}
\left(u_{i} u_{k}\right)=\frac{J I}{l^{2}}\left(U_{i} u_{k}-U_{k} u_{i}\right)- & \frac{I}{\Lambda l^{2}} M_{i k} \\
& =-\left(U_{i} U_{k}\right) \\
\left(u_{i} U_{k}\right)=-\frac{J I}{l^{2}}\left\{g_{i k}+\left(u_{i} u_{k}\right.\right. & \left.\left.-U_{i} U_{k}\right)\right\} \\
& =-\left(U_{i} u_{k}\right) .
\end{aligned}
$$

Endlich sind noch nach dem gleichen Verfahren zu bilden

$$
\begin{aligned}
\left(u^{l} M_{i k}\right) & =-I A\left\{\delta_{i} u_{k}-\delta_{k}^{l} u_{i}\right\} \\
\left(U^{l} y_{i k}\right) & =-T A\left\{\delta_{i}^{l} U_{k}-\delta_{k} U_{i}^{\prime}\right\} .
\end{aligned}
$$

Von besonderer Einfachheit und besonderem Interesse werden nun die $\mathrm{P}$ o is s o n-Klammern der Komponenten von $\mathfrak{b}$ und $\mathfrak{B}$. Wir haben $\mathbf{z u}^{-}$ nächst zu bilden

$$
\begin{aligned}
& \left(\frac{v_{i .}}{c} \frac{v_{k}}{c}\right)=\left(\frac{u_{i}}{u_{4}} \frac{u_{k}}{u_{4}}\right)= \\
& \frac{1}{\left(u_{4}\right)^{3}} \cdot\left\{u_{4}\left(u_{i} u_{k}\right)+u_{k}\left(u_{4} u_{i}\right)+u_{i}\left(u_{k} u_{4}\right)\right\}
\end{aligned}
$$

und

$$
\begin{gathered}
\left(\frac{V_{i}}{c} \frac{V_{k}}{c}\right)=\left(\frac{U_{i}}{U_{4}} \frac{u_{k}}{u_{4}}\right)=\frac{1}{\left(u_{4}\right)^{2}\left(U_{4}\right)^{2}}\left\{u_{4} U_{4}\left(U_{i} u_{k}\right)\right. \\
\left.-u_{k} U_{4}\left(U_{i} u_{4}\right)-U_{i} u_{4}\left(U_{4} u_{k}\right)+U_{i} u_{k}\left(U_{4} u_{4}\right)\right\} .
\end{gathered}
$$

Beim Einsetzen der Ausdrücke (5.7) heben sich die runden Klammern jeweils zyklisch heraus, und es bleibt, wenn man noch (5.2) heranzieht,

$$
\begin{aligned}
\left(\frac{V_{i}}{c} \frac{v_{k}}{c}\right) & =I \frac{J}{l^{2}} \frac{1}{u^{4} U^{4}}\left(\delta_{i k}-\frac{V_{i}}{c} \frac{v_{k}}{c}\right) \\
\left(\frac{v_{1}}{c} \frac{v_{2}}{c}\right) & =I \frac{I}{l^{2}} \frac{U^{4}}{\left(u^{4}\right)^{3}} \frac{V_{3}}{c} \\
\left(\frac{V_{1}}{c} \frac{V_{2}}{c}\right) & =-I \frac{I}{l^{2}} \frac{u^{4}}{\left(U^{4}\right)^{3}} \frac{v_{3}}{c} .
\end{aligned}
$$

Die erste dieser Formeln wiederholen wir der Deutlichkeit halber noch einmal für die Indices 1 und 2 unter Einführung von $u_{4}$ und $U_{4}$ nach (3.18) und (3.20) und formaler Symmetrisierung der rechten Seite:

$\left(V_{2} v_{1}\right)=$

$-\Gamma \frac{J}{l^{2}} \sqrt{\frac{V^{2}}{c^{2}}-1} \sqrt{1-\frac{v^{2}}{c^{2}}} \cdot \frac{1}{2}\left(V_{2} v_{1}+v_{1} V_{2}\right)$. 
Diese Poisson-Klammer bildet nämlich, im Rahmen der klassischen Theorie, die Auflösung des Widerspruchs, auf den wir am Schlusse der vorangehenden Arbeit geführt wurden, als wir Vertauschungsrelationen aufzustellen versuchten, die mit den Bewegungsgleichungen eines punktförmigen Teilchens unter dem Einflusse der Strahlungskraft vereinbar sein sollten. Wir fanden dort unter (31):

$i\left(v_{2} v_{1}-v_{1} v_{2}\right) \div \frac{3}{2 \gamma}\left(1-\frac{v^{2}}{c^{2}}\right) \frac{1}{2}\left(v_{2} v_{1}+v_{1} v_{2}\right)$.

Der Faktor $i$ entspricht hier der Vertauschungsrelation; $\gamma$ ist die Feinstrukturkonstante, und das Zeichen $\div$ bedeutet, daß die rechte und die linke Seite aus Gründen ihrer entgegengesetzten Symmetrie nicht einander gleichgesetzt werden konnten. Eine solche Symmetrieschwierigkeit kann in unsern durch einheitliche Differentiation hergeleiteten $\mathrm{P}$ o is s o n-Klammern natürlich niemals auftreten, und man sieht, daß sie sich unter Aufrechterhaltung der engsten Analogie hebt, wenn man die der versuchten Vertauschungsrelation entsprechende $\mathrm{P}$ o i s s o $\mathrm{n}$ - Klammer nicht mit den Komponenten der Geschwindigkeit unter sich, sondern mit ihnen und denen der ihnen nach (3.25) zugeordneten Geschwindigkeit bildet. Die Einführung der Momente erlaubt als̄o tatsächlich, diese fundamentale Schwierigkeit wenigstens im Bereich der Poisson-Klammern zu beheben. - Die Pois s o n-Klammern der $\mathfrak{b}$ - und $\mathfrak{B}$-Komponenten unter sich müssen natürlich wesentlich anders lauten als die früheren, widerspruchsvollen Vertauschungsrelationen.

Formel (5.12) gewinnt noch dadurch an Interesse, daß die Analogie mit (5.13) offenbar dann - und nur dann - besonders eng wird, wenn $\mathfrak{b}$ und $\mathfrak{B}$ möglichst gleich werden. Da sie durch die Bedingung (1.2) aneinander gebunden sind, bedeutet das, daß sie parallel und beide nahe gleich der Lichtgeschwindigkeit sein müssen, wobei dann $\mathfrak{B}$ ebensoweit $d \quad r$, wie $\mathfrak{b}$ darunter liegen muß. In diesem Falle stimmt auch das Produkt der Wurzeln in (5.12) mit dem Faktor $1-v^{2} / c^{2}$ der rechten Seite von (5.13) in erster Näherung überein. Nun geht die ganze Tendenz der Bewegung, wie wir schon in der ersten Arbeit zu diesem Gegenstande zeigten, dahin, die Geschwindigkeit sehr schnell der Lichtgeschwindig- keit entgegen wachsen zu lassen. Dies entspricht wieder ganz den Verhältnissen in der Dir a cschen Theorie, wo das Teilchen bekanntlich eine hochfrequente "Zitterbewegung“ mit Lichtgeschwindigkeit um den langsamer fortschreitenden Schwerpunkt ausführt. Die Aussicht, diesem noch so wenig geklärten Vorgange wenigstens mit einer klassischen Theorie näher zu kommen, wird leider dadurch getrübt, daß hier der Bewegungsablauf unübersehbar von den zahlreichen, willkürlichen Anfangsbedingungen der vielen Variablen abhängt. Hier müssen eben doch die Quantenbedingungen eingreifen. Ohne diese läßt sich auch der Faktor $\Gamma J / l^{2}$ in (5.12) nicht angeben. Er darf, auch wenn man (5.12) als Vertauschungsrelation versteht und einen Faktor $h$ ergänzt, nicht etwa durch den Faktor $3 / 2 \gamma$ von (5.13) ersetzt werden, weil $J$ und $l$ nicht mit allen Größen vertauschbar und daher keine Bewegungskonstanten sind. Jedenfalls entspricht er wegen der Kleinheit von $\gamma$ einem ,großen“ Faktor. d. h. die $v_{i}, V_{k}$ sind im wesentlichen antikommutativ.

\section{Hamilton-Funktion und Bewe- gungsgleichungen}

Nachdem wir uns in den vorangehenden $A b-$ schnitten ein System von relativistisch kovarianten $\mathrm{P}$ o i s s o n - Klammern verschafft haben, können wir nun nach Vorgabe einer $\mathrm{Hamilton-}$ schen Funktion $H$ nach der Formel

$$
\frac{d X}{d t}=(H X)
$$

für alle vorkommenden Größen $X$ ein System von ebenfalls kovarianten Bewegungsgleichungen ableiten. Wollen wir nach der Eigenzeit $\tau$ differentiieren, so haben wir nur gemäß

$$
d \tau=\sqrt{1-\frac{v^{2}}{c^{2}}} d t=\frac{d t}{u^{4}}
$$

alle Gleichungen mit $u^{4}$ zu multiplizieren. Am bequemsten schreiben sie sich mit Benutzung des Weltlinienelementes

$$
d s=c d \tau .
$$

Wir werden die Differentiation nach der Zeit 
durch einen Punkt, die nach dem Linienelement durch einen Strich andeuten ${ }^{16}$.

Die Konstruktion der Hamilton-Funktion verläuft $z$ wangläufig, wenn man weiterhin $\mathfrak{v}$ als Geschwindigkeit im gewöhnlichen Sinne auffaßt. Es muß dann für die Koordinaten $x^{i}(i=1,2,3)$ gelten

$$
\dot{x}^{i}=\left(H x^{i}\right)=\frac{\partial H}{\partial p_{i}}=v^{i} .
$$

Da $\mathfrak{p}$ und $\mathfrak{v}$ voneinander unabhängig sind, kann infolgedessen $H$ nur die Form py zuzüglich eines von $\mathfrak{p}$ unabhängigen Ausdruckes haben. Wegen seiner Bedeutung als einer Energie muß dann

$$
p_{\iota} u^{l}=\mathfrak{p} \mathfrak{u}+p_{4} u^{4}=-m c
$$

eine Invariante sein, wobei $m$ die Natur einer Masse hat. Daraus ergibt sich schon, daß H, soweit nur kinetische Energie in Frage kommt, die Form

$$
H=c p^{4}=-c p_{4}=\mathfrak{p} \mathfrak{v}+\frac{m c^{2}}{u^{4}}
$$

haben muß; das ist wieder die D i r a c sche Form. Die Einführung äußerer, durch ein Vektorpotential $\mathfrak{A}$ und ein skalares Potential $V$ gekennzeichneter Kräfte hat nun so zu erfolgen, daß bei Weglassen der Strahlungskraft die gewöhnlichen Bewegungsgleichungen einer Punktladung resultieren. Der vorangehenden Arbeit zufolge - Gl. (6) und $(8)^{5}$ - bleiben die der Koordinaten und Impulse von der Strahlungskraft überhaupt unbeeinflußt; ihre Einführung erfolgt in der gewohnten Weise dadurch, daß man $\mathfrak{p}$ und $p^{4}$ durch

$$
\begin{aligned}
\mathfrak{g} & =\mathfrak{p}+\frac{e}{c} \mathfrak{A} \\
g^{4} & =p^{4}+\frac{e}{c} V
\end{aligned}
$$

ersetzt. An Stelle der Gl. (6.5) tritt dann

$$
g_{\imath} u^{\imath}=-m c \text {. }
$$

Wir wollen auch, zunächst nur.der Abkürzung halber, für die mit den $U^{i}$ gebildete Invariante eine entsprechende Bezeichnung einführen durch

$$
g_{l} U^{l}=-M c \text {. }
$$

Die beiden Gleichungen sind ganz verschieden $\mathrm{zu}$

16 Man wolle einen mehrmaligen Wechsel der Bezeichnung entschuldigen: in der Zuschrift an ,die Naturwissenschaften" bezog sich der Punkt, in "der vorangehenden Arbeit der Strich auf die Eigenzeit. verstehen: (6.8) ist bei gegebenem $m$ eine Definitionsgleichung für $p_{4}$, umgekehrt (6.9)-bei gegebenem $p_{4}$ eine solche für $M$. Für $H=c p^{4}$ ergibt sich nun aus (6.8)

$$
H=\mathfrak{g} \mathfrak{v}-e V+\frac{m c^{2}}{u^{4}},
$$

woraus durch $\mathrm{P}$ oisson-Klammer-Bildung nach Impulsen in Übereinstimmung mit der vorangehenden Arbeit

$$
\dot{p}_{i}=\left(H p_{i}\right)=-\frac{\partial}{\partial x^{i}} e\left(\mathfrak{A} \frac{\mathfrak{v}}{c}-V\right)
$$

und weiter mit Einführung des Feldtensors $F_{i k}$ der äußeren Kräfte

$$
\dot{g}_{i}=-\frac{e}{c} F_{i k}^{\prime} v^{k}
$$

$\left(v^{4}=c\right)$ oder

$$
g_{i}^{\prime}=-\frac{e}{c} F_{i k} u^{k}
$$

hervorgehen; das sind die gewöhnlichen Gleichungen. Daß sie auch in der D i r a c schen Theorie gelten, wurde zum ersten Male von $\mathrm{F}$ o c k ${ }^{17}$ gezeigt.

Die $\mathfrak{v}, \mathfrak{M}, \mathfrak{P}$ sollten mit den Koordinaten und Impulsen vertauschbar sein. Sie sprechen dann bei der $\mathrm{P}$ oisson-Klammern-Bildung mit $H$ lediglich auf $\mathfrak{v}, u^{4}$ und $m$ an. Wir können nämlich noch zulassen, daß $m$ von den Invarianten $I$ und $J$, d.h. wegen der Konstanz von $I$ wesentlich nur von $J$, abhängt, und werden das natürlich tun, nachdem wir in der vorangehenden Arbeit die emittierte Energie in der Ruhmasse mitgezählt und diese dadurch zeitveränderlich gemacht haben.

Für die Ausrechnung der Bewegungsgleichungen nach (6.1) ist im vorangehenden Abschnitte alles zusammengestellt, so daß wir uns begnügen können, die Resultate anzugeben. Sie lauten

I. für die Invarianten:

$$
I^{\prime}=0 \quad J^{\prime}=\Gamma M c .
$$

II. für die Vierervektoren:

$$
\begin{aligned}
u_{i}^{\prime}=\frac{J \Gamma}{l^{2}}\left\{m c U_{i}-M c u_{i}\right. & \left.-\frac{1}{\Lambda J} g^{l} M_{l i}\right\} \\
& +\Gamma U_{i} \frac{\partial m c}{\partial J}
\end{aligned}
$$

$U_{i}^{\prime}=\frac{J I}{l^{2}}\left\{m c u_{i}-M_{c} U_{i}-g_{i}\right\}+\Gamma u_{i} \frac{\partial m c}{\partial J}$.

17 V. F o c k, Z. Physik 55, 127, [1929]. 
III. für den Momententensor:

$$
M_{i k}^{\prime}=-\Gamma \Lambda\left\{g_{i} u_{k}-g_{k} u_{i}\right\} .
$$

Die erste Gleichung (6.15) tritt also an Stelle der Gl. (7) der vorangehenden Arbeit, die in unsern jetzigen Bezeichnungen

$$
u_{i}^{\prime}=\frac{3 c}{2 e^{2}}\left\{\text { mc } u_{i}-g_{i}\right\}
$$

lautet; die zweite Gleichung (6.15) ist nur eine Folge,der ersten auf Grund von (5.2). Es ist aber bequem, die Gleichungen nebeneinander $\mathrm{zu}$ benutzen; (5.2) erscheint dann als ein partikuläres Integral. Wir fügen noch hinzu die Gleichung der gewöhnlichen Theorie ohne Strahlungskraft

$$
\text { m } c u_{\imath}=g_{i} .
$$

Ihr Bestehen ist bekanntlich wesentlich für das Bestehen des Drehimpulssatzes. In einem Zentralfelde wird $\mathfrak{g}=\mathfrak{p}$ und die Anderung von $\mathfrak{p}$ parallel dem Radiusvektor $\mathfrak{x}$, daher

$$
\frac{d[\mathfrak{r} \mathfrak{p}]}{d s}=[\mathfrak{u} \mathfrak{p}],
$$

und das verschwindet nach (6.18). Es verschwindet aber nicht nach (6.17), sondern geht über in

$$
\frac{d[\mathfrak{r} \mathfrak{p}]}{d s}=-\frac{2 e^{2}}{3 c}\left[\mathfrak{u} \mathfrak{u}^{\prime}\right]
$$

die Strahlungskraft verletzt den Drehimpulssatz. In unserer neuen Theorie tritt er aber wieder in Kraft für die Summe von Bahndrehimpuls und Spin, hier vertreten durch das negative magnetische Moment. In der Tat gilt nach (6.16) für $\mathfrak{U}=0, \mathfrak{g}=\mathfrak{p}$ :

$$
-\frac{d \mathfrak{M}}{d s}=\Gamma \Lambda[\mathfrak{p} \mathfrak{u}]
$$

daher ist jetzt, da (6.10) und (6.19) fortbestehen, nach (6.19) und (6.21)

$$
\mathfrak{j}=[\mathfrak{r} \mathfrak{p}]-\frac{\mathfrak{M}}{\Gamma \Lambda}
$$

zeitlich konstant. Dies Ergebnis ist unabhängig von der Form der Funktion $m(J)$.

7. Das Auftreten der Strahlungskraft

Unserem Plane gemäß hätten wir nun alle Veränderlichen bis auf die Lagenkoordinaten und ihre Ableitungen zu eliminieren und zu versuchen, die Funktion $m(J)$ und die Konstante $I$ so $z u$ bestimmen, daß in erster Näherung die Bewegungsgleichung für ein punktförmiges Teilchen mit der Strahlungskraft

$$
s_{i}=\frac{2 e^{2}}{3 c}\left(u_{i}^{\prime \prime}-u_{i}\left(u^{\prime}\right)^{2}\right)
$$

herauskommt. [Hier und weiterhin lassen wir bei Produkten von zwei Vierervektoren zur Abkürzung und leichteren Lesbarkeit die Indices weg, schreiben also $(u)^{2}$ und $g U$ statt $u_{\iota} u^{l}$ und $g_{\iota} U^{l}$ usw.] Wir haben dieses Problem der mathematischen Schwierigkeiten halber zunächst einmal für den Fall verschwindender äußerer Kräfte $\left(F_{i k}\right)$ durchgerechnet. Der Ausdruck für die Strahlungskraft muß auch dann bestehen bleiben, weil er von den $F_{i k}$ nicht direkt, sondern nur durch die $u^{\prime}, u^{\prime \prime}$ abhängt. Das Ergebnis ist, wie vorauszusehen war, daß man mit der einen Funktion $m(J)$ schon dann nicht alle Bedingungen erfüllen kann. Es müßten mindestens gewisse Größenordnungsbeziehungen erfüllt sein, über deren Zutreffen nur sehr eingehende Rechnungen mit Berücksichtigung der $F_{i k}$ Auskunft geben könnten. Nun ist nicht anzunehmen, daß sich die Lösung eines so grundsätzlichen Problems hinter solchen Komplikationen verbirgt. Wir möchten sie vielmehr in einer ganz anderen Richtung suchen. In der vorangehenden Arbeit wurde die Bewegung konservativ gemacht, indem man die Ruhmasse nach dem Gesetz

$$
m^{\prime}=\frac{2}{3} \frac{e^{2}}{c^{2}}\left(u^{\prime}\right)^{2}
$$

zunehmen ließ und in der Bewegungsgleichung $\left(m c u_{i}\right)^{\prime}$ statt $m_{c n}^{\prime} n_{i}^{\prime}$ schrieb. Dieser Kunstgriff war in Punkt 1 der Einleitung gemeint. In der vorliegenden Fassung der Theorie ist die Konstanz der Ha mil t o n - Funktion unabhängig davon gewahrt, weil die Bewegungsgleichungen aus ihr abgeleitet werden. Dafür haben wir jetzt durch das Vorhandensein zweier Geschwindigkeiten zwei Größen $m$ und $M$ von der Natur einer Masse, und es liegt nahe, $m$ wieder konstant $z u$ halten und dafür nach der Veränderlichkeit von $M z u$ fragen, in der Erwartung, die emittierte Energie dort wiederzufinden. Man hätte dann eine reinliche Scheidung zwischen Ruhmasse $m$ und Feldenergie $M c^{2}$. Konstanz von $m$ bedeutet, da $m$ nur über $J$ von der Zeit abhängt, $\partial m / \partial J=0$ in 
den Gl. (6.15), und nun besteht folgender $\mathrm{Zu}$ sammenhang: aus

$$
U_{i}^{\prime}=\frac{I}{J}\left\{m c u_{i}-M c U_{i}-g_{i}\right\}
$$

folgt einerseits, nach (6.8) und (6.9),

$$
g U^{\prime}=-\frac{\Gamma}{J}\left\{(m c)^{2}-(M c)^{2}+g^{2}\right\},
$$

anderseits aber auch wegen $u^{2}=-1, U^{2}=+1$, $u U=0$ nach den nämlichen Formeln

$$
\left(U^{\prime}\right)^{2}=\left(\frac{I}{J}\right)^{2}\left\{(m c)^{2}-(M c)^{2}+g^{2}\right\} ;
$$

folglich ist

$$
g U^{\prime}=-\frac{J}{T}\left(U^{\prime}\right)^{2}
$$

Nun ist nach der Definition (6.9)

$$
M^{\prime} c=-g U^{\prime}-g^{\prime} U,
$$

also nach (6.13) und (7.6)

$$
M^{\prime}=\frac{J}{I c}\left(U^{\prime}\right)^{2}+\frac{e}{c^{2}} F_{i k} U^{i} u^{k} .
$$

Das wäre gerade in Übereinstimmung mit (7.2), wenn $u, u^{\prime}$ an Stelle von $U, U^{\prime}$ stände (beachte $F_{i k} u^{i} u^{k}=0$ ) und

$$
J=\frac{2}{3} \frac{e^{2}}{c} I
$$

gesetzt werden könnte, und beides trifft bis zu einem gewissen Grade zu. Einerseits geht nämlich, wie schon am Schlusse des 5. Abschnittes bemerkt wurde, die Bewegungstendenz dahin, daß $\mathfrak{B}$ und $\mathfrak{b}$, daher auch $U_{i}$ und $u_{i}$ einander gleich werden. Allerdings geht das auf Kosten ihrer Endlichkeit, so daß der Grenzwert von $F_{i k} U^{i} u^{k}$ auch von Null verschieden sein könnte. Anderseits gilt für $J$ die Bewegungsgleichung (6.14)

$$
J^{\prime}=I^{\prime} M c .
$$

Wenn also $M$ von Null beginnen würde, so wäre wenigstens in der Anfangsphase $J=$ constans, wie (7.9) verlangt. Die Sache wäre dann so aufzufassen, daß die $\mathrm{H}$ a $\mathrm{m}$ i l t o n sche Funktion $H$ nicht die Energie des Teilchens, sondern eine passend als Termwert zu bezeichnende Größe wäre, während die Energie durch denselben Ausdruck mit $m-M$, also durch

$$
E=\mathfrak{g} \mathfrak{v}-e V+\frac{(m-M) c^{2}}{u^{4}}
$$

gegeben wäre, denn das geht im Ruhsystem des Teilchens $\left(\mathfrak{v}=0, u^{4}=1\right)$ bis auf Energie der Lage in $(m-M) c^{2}$ über.

Ein vollständiger Beweis dieser letzten Vermutungen wäre-wohl recht schwierig und als rein klassischer Tatbestand auch wenig weittragend. Es möge daher einstweilen genügen, festzustellen, daß wir mit der jetzigen, widerspruchsfreien Fassung der Theorie dem alten Ausgangspunkte jedenfalls sehr nahe geblieben sind. Die weiteren Überlegungen sollten sich vielmehr der Quantisierung zuwenden, womit sich auch die Konstante $\Gamma$ bestimmen müßte. Unsere Theorie ist durch ihre vollständige Begründung auf $\mathrm{Poisson-Klammern}$ ganz auf diesen Schritt hin angelegt. Die Verwandlung der Klammern in Vertauschungsrelationen erfordert aber wegen der nötigen Symmetrisierungen und anderer Verallgemeinerungen noch eine besondere Untersuchung. 